\title{
Challenges in the Study of Adjuvant Chemoradiation After Pancreaticoduodenectomy
}

\author{
Christopher H. Crane, $\mathrm{MD}^{1}$, Gauri R. Varadhachary, $\mathrm{MD}^{2}$, Robert A. Wolff, $\mathrm{MD}^{2}$, and Jason B. Fleming, $\mathrm{MD}^{3}$ \\ ${ }^{1}$ Department of Radiation Oncology, Unit 97, The University of Texas M. D. Anderson Cancer Center, Houston, TX; \\ ${ }^{2}$ Department of Gastrointestinal Oncology and Digestive Diseases, Unit 426, The University of Texas M. D. Anderson \\ Cancer Center, Houston, TX; ${ }^{3}$ Department of Surgical Oncology, Unit 444, The University of Texas M. D. Anderson \\ Cancer Center, Houston, TX
}

Inadequate preoperative staging, high rates of incomplete tumor resection, and early distant tumor dissemination make the study of adjuvant therapy in pancreatic adenocarcinoma particularly challenging. To understand the value of chemoradiation in an adjuvant study, one needs to confirm that patients accrued to the study have undergone a potentially curative resection. This is of critical importance because patients left with a positive surgical margin have a median survival of less than 12-14 months, a result that may be achieved by nonsurgical therapies. ${ }^{1}$ Surprisingly, even highvolume university-based hospitals report positive surgical margins as high as $50 \% .^{2,3}$ The frequency of positive surgical margins is probably much higher in lower-volume centers. Strict assessments of surgical margins with particular attention to the retroperitoneal margin (also known as the SMA margin or uncinate margin) have not been widely adopted, leading to underreporting of the true margin positive rate in some studies. Furthermore, the critical distinction between potentially curative (R1) resection and noncurative gross residual disease ( $\mathrm{R} 2$ resection) cannot be made through pathologic examination alone. This was reflected in the Radiation Therapy Oncology Group (RTOG) 97-04 trial, where the surgical margin status for approximately $25 \%$ of enrolled patients was not reported in the operative note or pathology report, and $\mathrm{R} 1$ versus $\mathrm{R} 2$ resection status could not be retrospectively determined. ${ }^{4}$ A similar disparity was observed in the European Study Group for Pancreatic Cancer (ESPAC-1) and Charite Onkologie (CONKO-001) trials. ${ }^{5,6}$ Although the proportion of patients having a

(C) Society of Surgical Oncology 2009

Published Online: 11 December 2009

C. H. Crane, MD

e-mail: ccrane@mdanderson.org positive surgical margin was quite low, local failure rates as a component of failure were high, ranging from $35 \%$ to $62 \%$. These local failure rates imply that a substantial proportion of patients receiving adjuvant therapy actually had incomplete, noncurative (R2) surgical resections and the "adjuvant" therapy they received after surgery actually served as treatment for incompletely resected locally advanced disease.

Subsets of pancreatic cancer patients also have rapid early tumor dissemination. The frequency of early distant relapse after pancreatectomy may be extrapolated from prospective trials of neoadjuvant therapy. Studies from the University of Texas M. D. Anderson Cancer Center, the Eastern Cooperative Oncology Group, and Duke University Medical Center have shown that approximately 15-20\% of patients with no evidence of distant disease on initial staging who enroll onto preoperative trials will develop radiographic evidence of metastatic disease within several weeks, as documented on their posttreatment preoperative restaging computed tomographic scan. ${ }^{7-9}$ Because computed tomography was not required before protocol entry in the early trials that evaluated the role of radiotherapy, patients undergoing $\mathrm{R} 2$ resection or who had rapidly progressive metastatic disease during the postoperative recovery period (15-20\% of patients) were not excluded from protocol entry. ${ }^{5,10,11}$ The failure to exclude these incurable patients from all three early trials evaluating chemoradiation diluted the statistical power and confounded the interpretation of the results because the number of incurable patients may not have been balanced between the arms. ${ }^{5,10,11}$ Even though it is naive to accept their conclusions at face value, these early trials are considered the best evidence so far to evaluate the role of radiotherapy in resected pancreatic cancer, but are they 
really? Because our ability to interpret the available phase III evidence is limited, the value of retrospective evidence with high surgical and pathologic quality control likely results in more homogenous group of patients who are more relevant to assess the benefit of radiotherapy. This may not be true in other tumor sites where the quality of the phase III evidence is better.

Evidence in support of the role of chemoradiation has come from retrospective studies from high-volume centers of surgical excellence such as the Mayo Clinic and the Johns Hopkins School (JHU) of Medicine. ${ }^{12,13}$ Like all retrospective single-arm studies, they are all confounded by selection bias that limits their interpretation and impact. Nevertheless, the surgical quality, perioperative care, and expertise in pathologic specimen processing and evaluation are excellent and help to eliminate those variables as sources of bias. However, because approximately $40 \%$ of patients in the Mayo series and 30\% in the JHU series did not receive adjuvant chemoradiation, there could be unidentified sources of bias that could have confounded the results. The study by Hsu et al. in this issue of the Annals of Surgical Oncology reports the pooled data from JHU and the Mayo Clinic and represents the first study that attempts to control for objective sources of bias using a matchedpair analysis. ${ }^{14}$ This is a large retrospective series that is well powered for a multivariate analysis. The analysis identifies younger age, the presence of positive surgical margins, and high-grade histology as factors associated with a higher probability of undergoing postoperative chemoradiation. Some of these factors, such as high-grade histology and positive surgical margins status, were also found to be independently associated with an up to $45 \%$ increased the risk of death. A unique strength of this analysis is the matched pairing, which is arguably the best attempt to retrospectively control for identifiable sources of selection bias in the delivery of adjuvant therapy in pancreatic cancer. With a large sample size (248 patients in each group), the authors were able to control for several objective variables in patient selection. Factors that were statistically significant on multivariate analysis (age, stage, differentiation, margin status, and nodal status) were matched between the treatment groups. However, as the authors point out, it was not possible from their data to control for clinical sources of bias such as performance status, poor nutritional status, prolonged postoperative recovery, and medical comorbidites. Declining performance status in particular may be a specific early clinical indicator of the onset or progression of radiographically occult metastatic disease. Astute clinicians would not generally recommend initiation of chemoradiation to patients who are in a state of clinical decline; this phenomenon in particular could have confounded the findings of the analysis.
The development of more effective systemic therapies is critical to improving surgical outcomes. However, the accumulated data clearly demonstrate local control as necessary for long-term survival. Thus, our ability to detect a benefit from therapies delivered in future clinical trials depends on our ability to select patients in whom the natural history of their disease can be altered. This is limited to patients undergoing a strictly assessed R0 resection who do not have metastatic disease at the time of protocol entry. Steps in this direction have been made in the more recent trials. The CONKO-1 and RTOG 97-04 trials both required postoperative preenrollment computed tomographic scans and excluded patients with clear evidence of residual local tumor or distant metastases. ${ }^{6,15}$ The results support the use of gemcitabine after pancreatectomy as a standard and have made an impact on clinical practice today.

It is impossible from phase III trials to ascertain what value, if any, radiotherapy adds to the treatment of appropriately selected patients who have undergone R0 pancreatic resection. One of the strengths of the current study is uniform surgical quality. On the other hand, even though the authors controlled for known prognostic factors in the analysis, potentially adverse prognostic factors such as declining performance status could not be controlled for. Even more homogeneous populations can be accrued with adherence to standardized definitions of resectability on the basis of high-quality preoperative radiographic imaging, accurate pathologic specimen processing, and surgical quality control. Effective quality control is extraordinarily challenging, particularly in the context of adjuvant trials where patients are traditionally enrolled weeks after the surgery and pathologic analysis has already been completed. The ESPAC-3 and European Organization for the Research and Treatment of Cancer (EORTC) 40013 postoperative trials both mandated the acquisition of highquality preoperative imaging, but did not require adherence to a standardized definition of resectability. Prospective surgical, pathologic, and radiotherapy quality assurance are planned in the RTOG/EORTC/Intergroup 0848 postoperative adjuvant trial. Surgeons will be required to have documented total gross excision of the tumor, and the status of all relevant surgical margins will need to be reported as part of the inclusion criteria. Preoperative imaging will be reviewed but not used as part of the inclusion criteria. Prospective radiographic, surgical, and pathologic quality control are also planned in the American College of Surgeons Oncology Group neoadjuvant trial, Z5041. This trial includes protocol-specified central imaging review of pretreatment and preoperative imaging, protocol-defined surgical technique, standardized assessment of the pathology specimen, and templated pathology reporting. Deviations are communicated back to the participating surgeon. These quality assurance efforts require a 
tremendous amount of work from the investigators and research staff, but the benefits could go beyond the accuracy of interpretation of future trials. For instance, confidential verbal or written feedback could be informative enough to influence practice standards among the participants. This may ultimately have a greater impact on patient survival than any specific adjuvant therapy.

Should this study change practice? This study may lead the group of clinicians who never recommend chemoradiation because of an underappreciation of the flaws of the completed phase III trials to reconsider the selected use of chemoradiation, especially after a period of chemotherapy. This strategy is reflected in the design of RTOG/EORTC/ Intergroup 0848, which includes gemcitabine-based chemotherapy to all patients for five cycles, followed by restaging and subsequent 1:1 randomization to chemoradiation or one additional cycle of chemotherapy. Outside enrollment in such a clinical trial, initial treatment should be systemic gemcitabine for 4 to 6 months followed by restaging and consideration of fluorouracil-based chemoradiation, particularly for patients with close or microscopically positive retroperitoneal margins.

\section{REFERENCES}

1. Sohn TA, Yeo CJ, Cameron JL, et al. Resected adenocarcinoma of the Pancreas-616 patients: results, outcomes, and prognostic indicators. J Gastrointest Surg. 2000;4:567-79.

2. Takai S, Satoi S, Toyokawa H, et al. Clinicopathologic evaluation after resection for ductal adenocarcinoma of the pancreas: a retrospective, single-institution experience. Pancreas. 2003;26:243-9.

3. Richter A, Niedergethmann M, Sturm JW, et al. Long-term results of partial pancreaticoduodenectomy for ductal adenocarcinoma of the pancreatic head: 25-year experience. World J Surg. 2003;27:324-9.

4. Regine W, Winter K, Abrams R, et al. RTOG 9704 a phase III study of adjuvant pre and post chemoradiation (CRT) 5-FU vs. gemcitabine $(\mathrm{G})$ for resected pancreatic adenocarcinoma. J Clin Oncol. 2006;24:4007.

5. Neoptolemos JP, Stocken DD, Friess H, et al. A randomized trial of chemoradiotherapy and chemotherapy after resection of pancreatic cancer. $N$ Engl J Med. 2004;350:1200-10.

6. Oettle H, Post $\mathrm{S}$, Neuhaus $\mathrm{P}$, et al. Adjuvant chemotherapy with gemcitabine vs observation in patients undergoing curative-intent resection of pancreatic cancer: a randomized controlled trial. JAMA. 2007;297:267-77.

7. Hoffman JP, Lipsitz S, Pisansky T, et al. Phase II trial of preoperative radiation therapy and chemotherapy for patients with localized, resectable adenocarcinoma of the pancreas: an Eastern Cooperative Oncology Group Study. J Clin Oncol. 1998;16:317-23.

8. White RR, Hurwitz HI, Morse MA, et al. Neoadjuvant chemoradiation for localized adenocarcinoma of the pancreas. Ann Surg Oncol. 2001;8:758-65.

9. Wolff RA, Evans DB, Crane $\mathrm{CH}$, et al. Initial results of a preoperative gemcitabine (GEM)-based chemoradiation for resectable pancreatic adenocarcinoma (abstract). Proc Am Soc Clin Oncol. 2002;21:130a.

10. Further evidence of effective adjuvant combined radiation and chemotherapy following curative resection of pancreatic cancer. Gastrointestinal Tumor Study Group. Cancer. 1987;59:2006-10.

11. Klinkenbijl JH, Jeekel J, Sahmoud T, et al. Adjuvant radiotherapy and 5-fluorouracil after curative resection of cancer of the pancreas and periampullary region: phase III trial of the EORTC gastrointestinal tract cancer cooperative group. Ann Surg. 1999;230: 776-82.

12. Corsini MM, Miller RC, Haddock MG, et al. Adjuvant radiotherapy and chemotherapy for pancreatic carcinoma: the Mayo Clinic experience (1975-2005). J Clin Oncol. 2008;26:3511-6.

13. Yeo CJ, Abrams RA, Grochow LB, et al. Pancreaticoduodenectomy for pancreatic adenocarcinoma: postoperative adjuvant chemoradiation improves survival. A prospective, single-institution experience. Ann Surg. 1997;225:621-33.

14. Hsu CC, Herman JM, Corsini MM, et al. Adjuvant chemoradiation for pancreatic adenocarcinoma: the Johns Hopkins HospitalMayo Clinic Collaborative Study. Ann Surg Oncol. (in press). DOI:10.1245/s10434-009-0743-7.

15. Regine WF, Winter KA, Abrams RA, et al. Fluorouracil vs gemcitabine chemotherapy before and after fluorouracil-based chemoradiation following resection of pancreatic adenocarcinoma: a randomized controlled trial. JAMA. 2008;299:1019-26. 